\title{
Low SAR planar antenna for multi standard cellular phones
}

\author{
M. Ben Ahmed ${ }^{1}$, M. Bouhorma ${ }^{1}$, F. Elouaai ${ }^{2}$, and A. Mamouni ${ }^{3, a}$ \\ 1 Computing and Telecommunications Research Group, Faculty of Science and Technology, BP 416, Tangier, Morocco \\ 2 Biotechnology and pathology laboratory Faculty of Science and Technology, BP 416, Tangier, Morocco \\ 3 DHS/MITEC IEMN, UMR-CNRS, Lille, France
}

Received: 5 February 2010 / Accepted: 15 December 2010

Published online: 22 February 2011 - (c) EDP Sciences

\begin{abstract}
In this paper the design of a multiband compact antenna for the integration into the new multi function mobile phones is presented. The antenna is matched to operate at GSM $920 \mathrm{MHz}$, WI-Fi $2.4 \mathrm{GHz}$ and HiperLan 5.1 GHz standards with low SAR levels. Return loss coefficient and radiation pattern of this antenna are computed in free space as well as in the presence of head. The specific absorption rate (SAR) of the planar antenna is calculated and compared with that of the monopole antenna. To examine the performance of this antenna, a prototype was designed, fabricated and measured; the simulation analysis was performed using the HFSS software, good agreement with the simulation providing validation of the design procedure.
\end{abstract}

\section{Introduction}

Growing consumer demand for multifunctional mobile handsets has seen an increase in the development of small multi-band antennas. It seems to be highly desirable to develop lightweight, low-profile and single feed mobile antenna, which can be used simultaneously in different frequency ranges and for different mobile services. The ability to cover a number of communication bands with one small antenna benefits both the end user and the manufacturer as it reduces the cost and complexity of the antenna system and allows more versatile function of a compact handset. In addition, the handset can be made more attractive to the consumer by reducing the radiation absorbed in the head, i.e. the specific absorption rate (SAR).

Internal planar antennas are very suitable in such applications since they are compact, low profile and easy to manufacture [1]. Starting with a main shorted resonator, several techniques must be simultaneously applied to achieve multiband performances: addition of parasitic shorted patches and capacitive loads, and use of slots.

Interaction of handset antennas with human head is a great consideration in cellular communications. The user's head influence on the antenna radiation pattern. Furthermore, thermal effect, when tissues exposed to unlimited electromagnetic energy, can be a serious health hazard. So standard organizations have set exposure limits in terms of the specific absorption rate (SAR) [2,3].

In this paper, a new miniaturized multiband antenna which supports GSM, WI-FI and HIPERLAN communication standard is proposed. In order to achieve the ob-

\footnotetext{
a e-mail: ahmed.mamouni@iemn.univ-lille1.fr
}

jective of designing a multiband patch antenna, we have begun with a simple model of patch which resonates on the HIPERLAN band frequency. Later we have studied the effect of changing the geometry by adding slots on the surface of the patch and finally, the impact of adding a resonator on the ground plane. Consequently we have deduced their influence on the resonance of the antenna on other frequency bands. The final geometry of the presented antenna is evaluated in the presence of the head model. In addition, the SAR quantity is computed for this antenna.

As pointed before, all simulations are performed using the Ansoft HFSS software.

\section{Antenna design and results discussion}

The parametric study is carried out to optimize the antenna structure and provide more information about the effects of the essential design parameters. The antenna performance is mainly affected by geometrical parameters such as adding slots and parasitic resonator.

\subsection{Basic model}

As starting model Figure 1 shows three dimensional views of the proposed antenna. The antenna is fed by a $50 \Omega$ microstrip line. The antenna and the partial ground plane are etched on opposite sides of Fr4-Epoxy substrate with a thickness of $1.6 \mathrm{~mm}$ and a relative permittivity $\varepsilon_{r}=4.4$. The substrate size of the proposed antenna is $40 \times 25 \mathrm{~mm}^{2}$. 


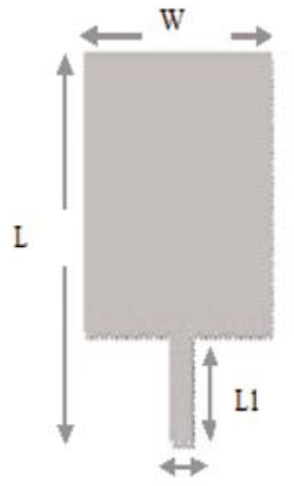

W1
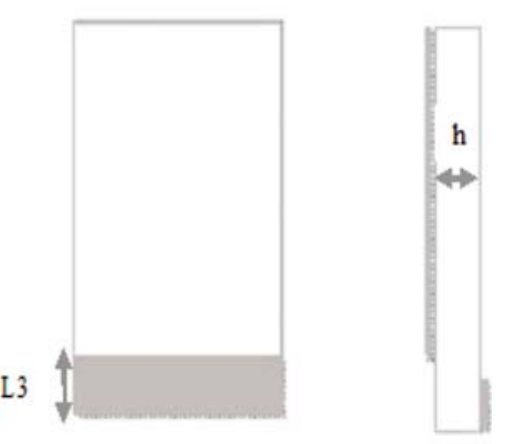

Fig. 1. Geometry of the proposed basic antenna.

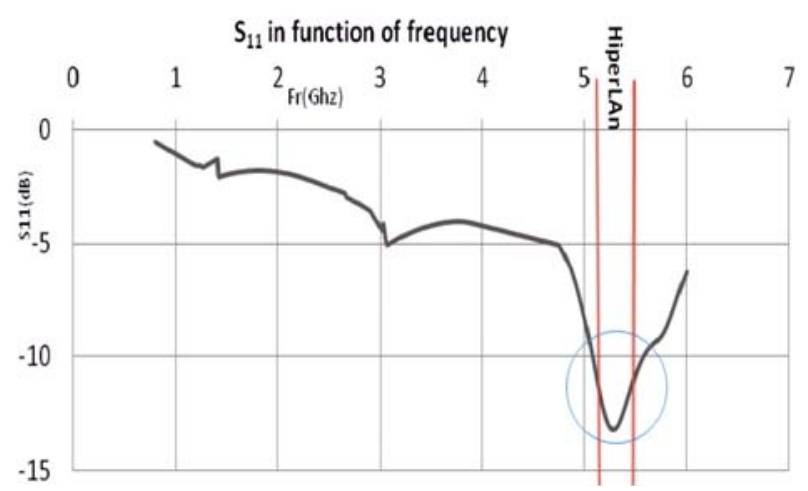

Fig. 2. (Color online) Return loss coefficient as a function of frequency.

As shown Figure 1, the radiating structure has dimensions of $L=25 \mathrm{~mm}$ and width of the patch $W=18 \mathrm{~mm}$ that is calculated using the microstrip calculation equations (1) and (2) to center the resonant frequency near the HiperLan band. As well as $L 2=8 \mathrm{~mm}$ and $W 2=3 \mathrm{~mm}$ that have been adjusted to conduct the input impedance to $50 \Omega$. Several studies $[4,5]$ have demonstrated that the use of a partial ground plane can widen the bandwidth of resonance and obtain a wideband antenna. The length of partial ground plane is optimized to $L 3=3 \mathrm{~mm}$.

$$
\begin{aligned}
W & =\frac{c}{2 f_{0}} \sqrt{\frac{2}{\varepsilon_{r}+1}} \\
L & =\frac{c}{2 f_{0} \sqrt{\varepsilon_{r}}}-2 \Delta L \\
\varepsilon_{\text {eff }} & =\left(\frac{\varepsilon_{r}+1}{2}\right)+\left(\frac{\varepsilon_{r}-1}{2}\right)\left(1+\left(10 \frac{h}{W}\right)^{-\frac{1}{2}}\right) \\
\Delta L & =0.412 h\left[\frac{\left(\varepsilon_{\text {eff }}+0.3\right)\left(\frac{W}{h}+0.264\right)}{\left(\varepsilon_{\text {eff }}-0.258\right)\left(\frac{W}{h}+0.813\right)}\right] .
\end{aligned}
$$

Figure 2 shows the simulated return loss coefficient, as we can see; the antenna basic model presents a good resonance in the HiperLan band.
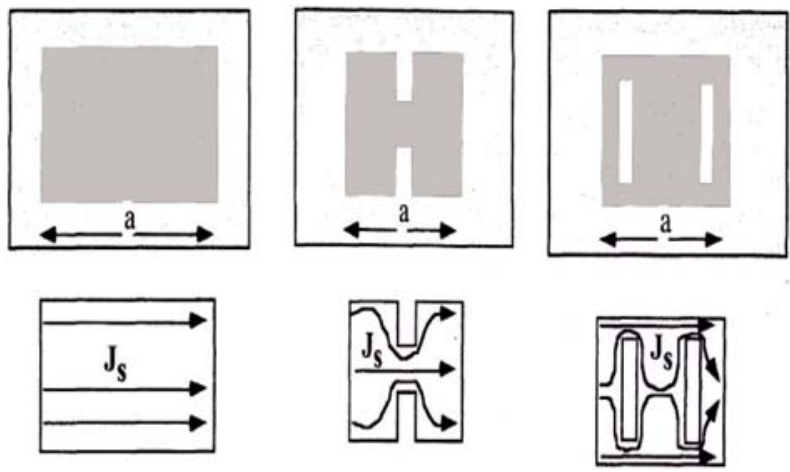

Fig. 3. Effects of adding slots.

\subsection{Variation of the antenna electrical length}

One of the objectives of this study is to lead the antenna to resonate at the GSM operating frequency. Additionally, the available cellular phones equipments at this frequency are now smaller and cheaper. However, the antenna at this frequency is relatively large $(33 \mathrm{~cm}$ at half-wavelength in free space) due to low operating frequency.

The increment of electrical length with the small antenna size is one of the popular techniques. This technique is done by changing the antenna geometry that's shown in Figure 3 and is not complicated for fabrication.

These slots force the surface currents to meander, thus artificially increasing the antenna's electrical length without modifying its global dimensions, which results a decrease in resonant frequency.

In our case, we have done a parametric simulation, we adjusted the position of the two slots and we calculated their lengths to resonate the antenna in the GSM band.

In Figure 4b we can see the new extension of the electrical length created by the addition of slots.

From the curve in Figure 5, we can clearly infer the effect of changing the patch geometry by adding the cracks which lead to a frequency decrease into the GSM band.

\subsection{Adding a parasitic resonator in the ground plane}

Previous studies demonstrated that the addition of a resonator in the ground plane causes the creation of resonance frequencies [6,7]. The Wi-Fi band resonance operation is achieved by combining one driven patch (Fig. 6, top) and one parasitic patch of the antenna (Fig. 6, bottom).

The dimensions of the resonator have been optimized as well as his location to show the Wi-Fi band, Table 1 presents the final antenna dimensions.

In order to practically realize an antenna, a standard FR-4 substrate is graved using a modern high resolution milling for cheap prototype production. The return loss coefficient of the microstrip antenna with the parasitic element was measured using a HP $8720 \mathrm{C}$ network analyzer. Figure 7 shows the comparison between the measured and simulated return loss coefficient as a function of frequency. 


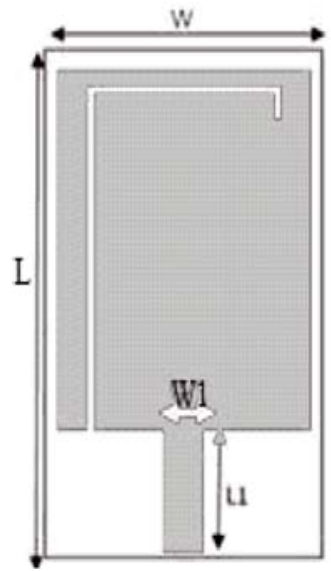

(a)

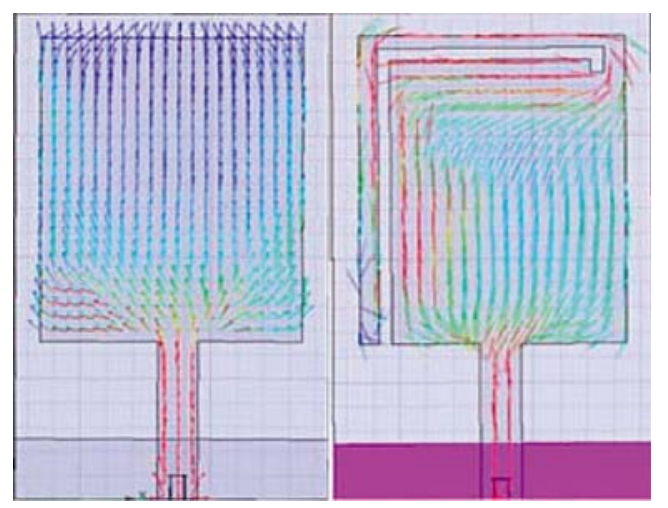

(b)

Fig. 4. (Color online) (a) Geometry of antenna after adding slots and (b) antenna current distribution before and after addition of slots.

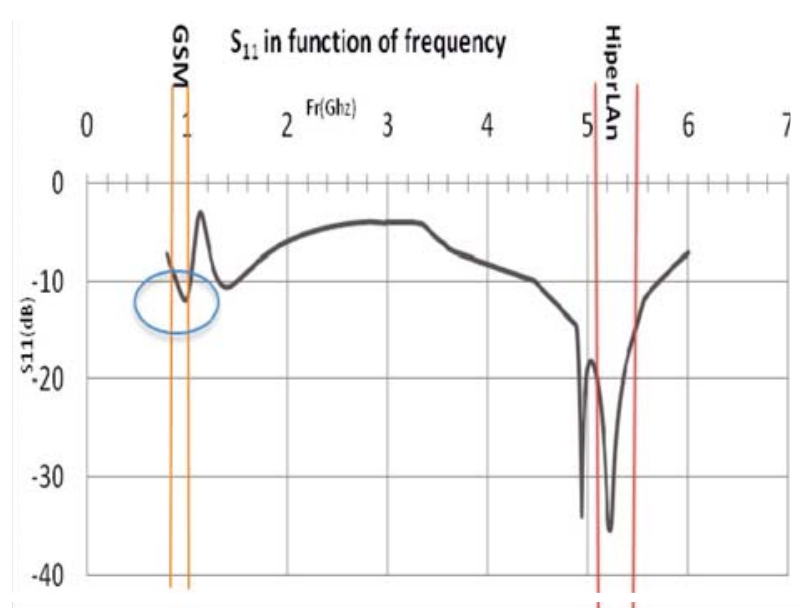

Fig. 5. (Color online) Return loss coefficient as a function of frequency.

As shown in Figure 7, it can be clearly seen that the addition of resonator adds another band resonance it's the Wi-Fi band. Hence we have arrived to satisfy the objective and obtain a multiband patch antenna which supports the standards of GSM, WI-FI and HiperLan. The simulated results are in good agreement with the measured results.

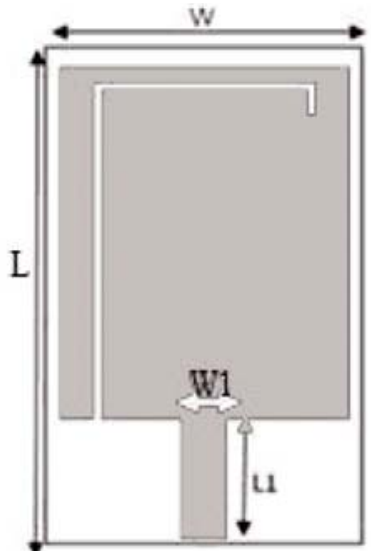

Top

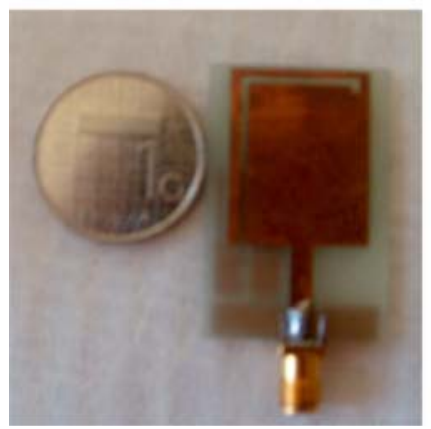

Fig. 6. (Color online) Geometry of the proposed final antenna.

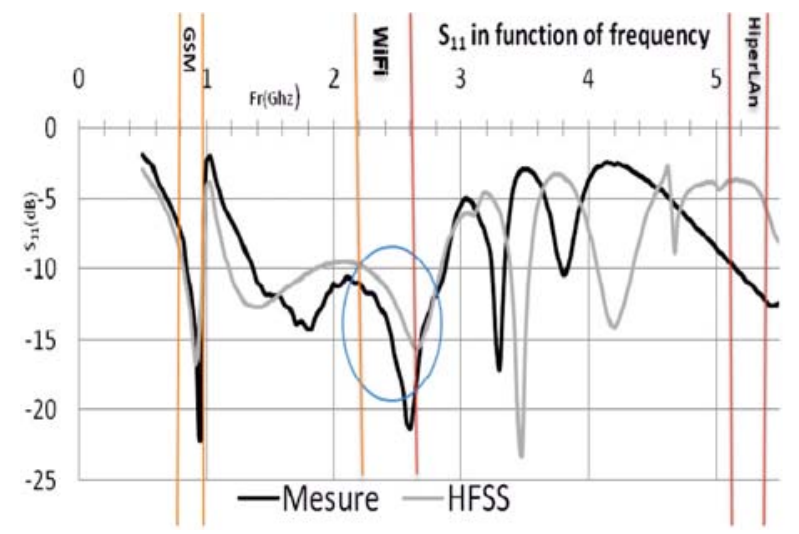

Fig. 7. (Color online) Return loss coefficient as a function of frequency.

Table 2 presents the frequency bands of the final planar antenna (Fig. 6).

The measured fractional bandwidths of double sided planar antenna were $7.05 \%$ for the lower band, $44 \%$ for the middle band and $11 \%$ for the upper band. In all cases the antenna offers sufficient bandwidth to cover all the European wireless systems.

Figure 8 shows the measured radiations patterns at $920 \mathrm{MHz}, 2.40 \mathrm{GHz}$ and $5.1 \mathrm{GHz}$.

The cuts of the radiation patterns of the studied antenna are shown in Figure 8. The radiations patterns are 


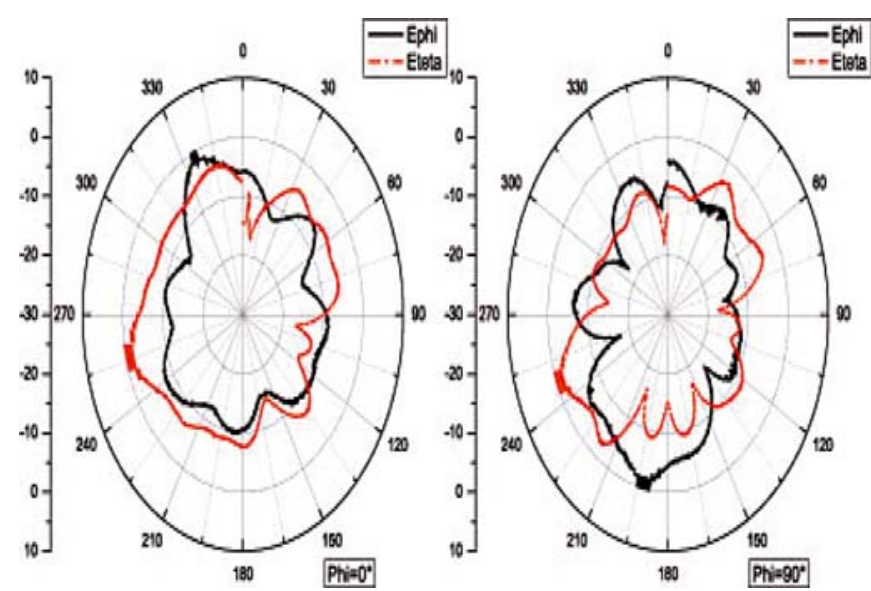

(a)

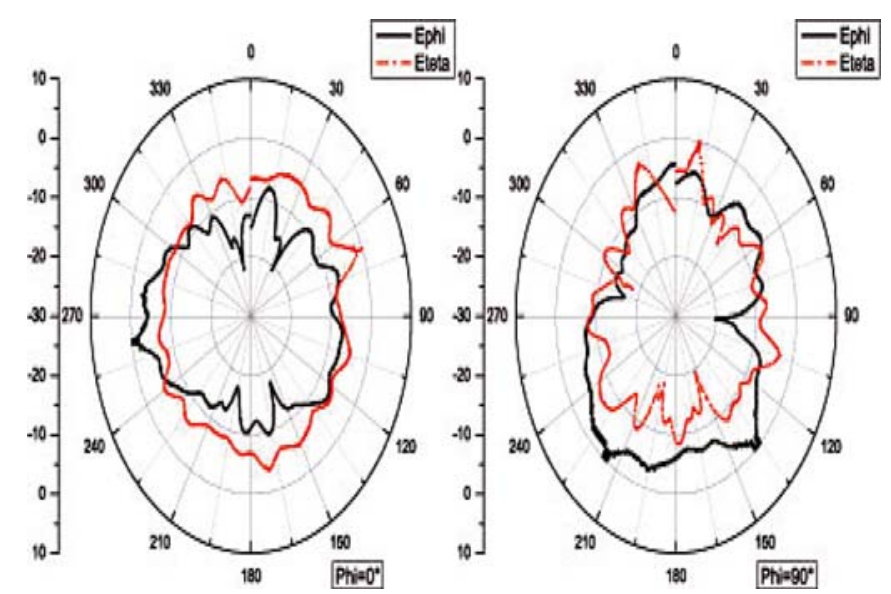

(b)

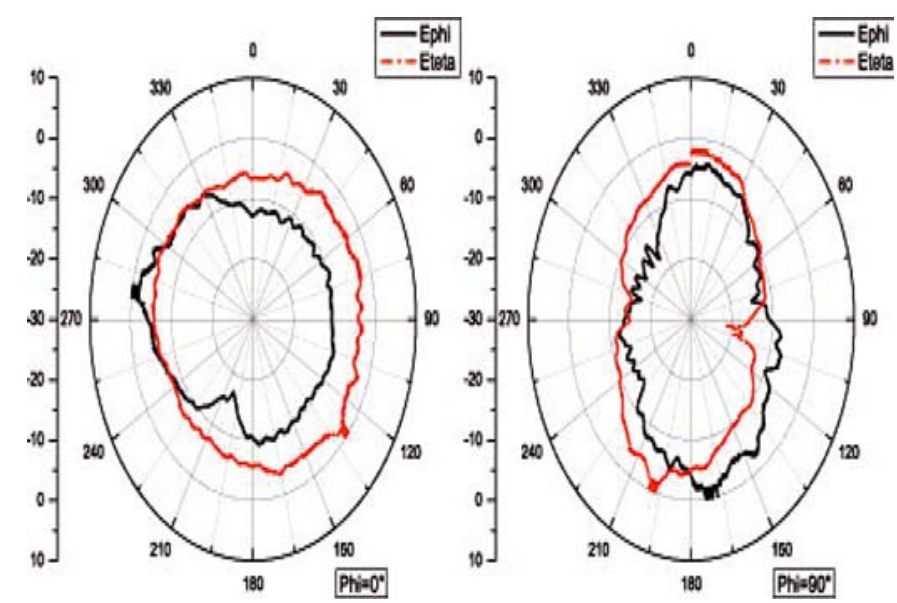

(c)

Fig. 8. (Color online) (a) Antenna $2 \mathrm{D}$ radiation pattern at $F=0.92 \mathrm{GHz}$, (b) antenna $2 \mathrm{D}$ radiation pattern at $F=2.4 \mathrm{GHz}$ and (c) antenna 2D radiation pattern at $F=5.1 \mathrm{GHz}$.

almost omni directional and resemble that of a half-wave dipole.

Table 3 shows that the lowest band GSM has the greater antenna gain of about $3 \mathrm{dBi}$.

\section{The antenna dosimetry}

The dosimetric system is composed by a phantom and a mobile phone. The mobile phone is modelled by a simple metal plate presented the phone chassis which is equipped in first place by a monopole antenna and secondly by the multi band patch antenna. The chassis dimensions in the design phase were roughly $120 \mathrm{~mm} 55 \mathrm{~mm} 20 \mathrm{~mm}$ (length width thickness), representing the typical dimensions of a modern mobile handset chassis [8].

The phone models were positioned beside the head according to the intended use position specified by CENELEC $[9,10]$. The phantom represents a human head filled with The spherical model had a uniform content at its core (representing the human brain) and the core was surround by two spherical shells representing the skull (bone)
Table 1. Antenna size details.

\begin{tabular}{cccc}
\hline Parameter & Value & Parameter & Value \\
\hline$L$ & $25 \mathrm{~mm}$ & $W$ & $18 \mathrm{~mm}$ \\
$L 1$ & $8 \mathrm{~mm}$ & $W 1$ & $3 \mathrm{~mm}$ \\
$L 3$ & $3 \mathrm{~mm}$ & $W r$ & $10 \mathrm{~mm}$ \\
$L r$ & $20 \mathrm{~mm}$ & $S$ & $16 \mathrm{~mm}$ \\
\hline
\end{tabular}

Table 2. Frequency and bandwidth.

\begin{tabular}{ccc}
\hline Frequency band & $\begin{array}{c}\text { Bandwidth } \\
(\mathrm{MHz})\end{array}$ & $\begin{array}{c}\text { Percentage } \\
\text { bandwidth }\end{array}$ \\
\hline $860-980$ & 120 & 7.05 \\
$1230-1950$ & 620 & 36.47 \\
$2100-2860$ & 760 & 44.70 \\
$5100-5300$ & 200 & 11.76 \\
\hline
\end{tabular}

and the muscle and the skin (skin) with their respective electromagnetic properties. The properties of the materials used in the simulations at $920 \mathrm{MHz}$ are presented in Table 4 [11]. 

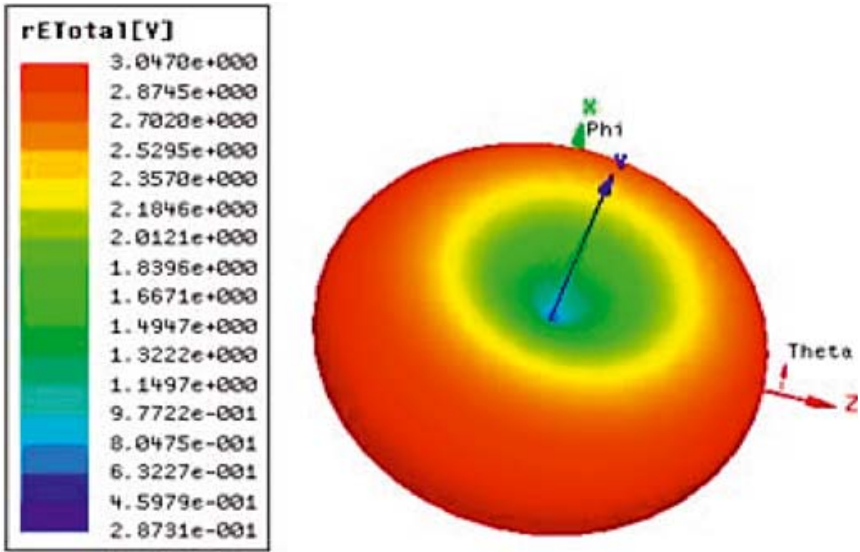

(a)
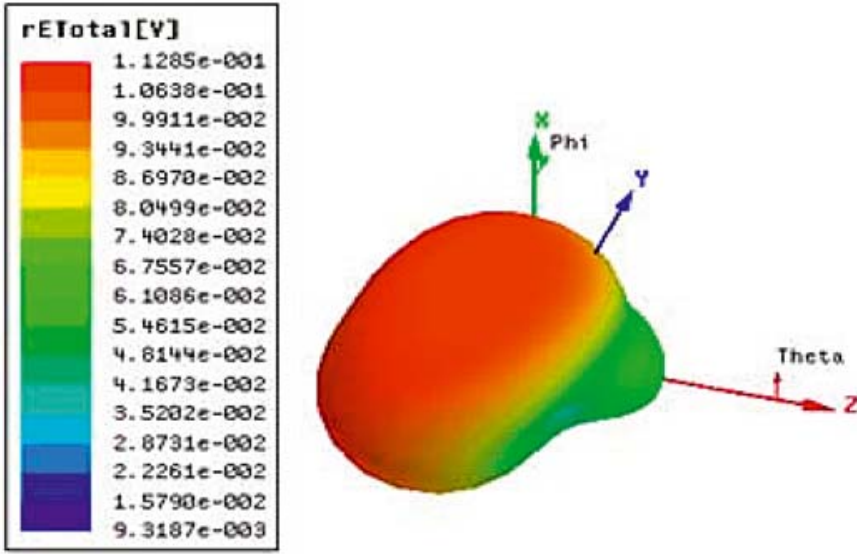

(b)

Fig. 9. (Color online) (a) Antenna 3D radiation pattern in a vacuum at $920 \mathrm{MHz}$ and (b) antenna 3D radiation pattern in the presence of phantom at $920 \mathrm{MHz}$.

Table 3. Gain of measured frequencies.

\begin{tabular}{cccc}
\hline Frequency & $920 \mathrm{MHz}$ & $2.4 \mathrm{GHz}$ & $5.1 \mathrm{GHz}$ \\
\hline Gain $(\mathrm{dBi})$ & 2.7 & 2.2 & 1.7 \\
\hline
\end{tabular}

Table 4. Phantom tissues dielectric characteristics in $920 \mathrm{MHz}$

\begin{tabular}{lcc}
\hline & Relative permittivity & Conductivity $(\mathrm{S} / \mathrm{m})$ \\
\hline DRY SKIN & 41.4 & 0.867 \\
BONE & 16.62 & 0.242 \\
BRAIN & 45.8 & 0.766 \\
\hline
\end{tabular}

The simulation approach consists on measuring and comparing the level of SAR absorbed by the phantom in the case of a classical phone model and the case of a phone composed by the new multiband patch antenna when a mobile phone emits at maximum power. We also made a comparison between the radiation pattern of our antenna in a vacuum and in the presence of phantom.

In mobile certification, the SAR is defined as the power absorbed by the unit of mass of tissue $(\mathrm{W} / \mathrm{kg})$ and is deduced from electric field values using the following equation:

$$
\mathrm{SAR}=\frac{d}{d t}\left(\frac{d W}{d m}\right)=\frac{\sigma|E|^{2}}{2 \rho} .
$$

Where $E$ is the total electric field in $\mathrm{V} / \mathrm{m}, \sigma$ is the conductivity in mhos $/ \mathrm{m}$, and $\rho$ the density of the tissue in $\mathrm{kg} / \mathrm{m}^{3}$. SAR is calculated as a function of position from the estimates of local fields and tissues properties. An integral of SAR over a volume of tissue containing a given mass gives the absorbed power. This is typically expressed in units of $\mathrm{W} / \mathrm{kg}$, or $\mathrm{mW} / \mathrm{cm}^{3}$ (for a given tissue density) averaged over $1 \mathrm{~g}$ of tissue.

Numerical studies have shown that the tissues of the ear region are most exposed, and a large part of the ab-
Table 5. SAR peaks in head tree layers model comparison between monopole and presented patch antenna.

\begin{tabular}{lcccc}
\hline Antenna & \multicolumn{3}{c}{ SAR (W/kg) } & $F(\mathrm{GHz})$ \\
& Skin & Bone & Brain & \\
\hline Monopole & 9.9 & 2.9 & 5.4 & \multirow{2}{*}{0.9} \\
Patch & 1.6 & 0.3 & 0.4 & \\
Monopole & 7.4 & 2.1 & 3.2 & \multirow{2}{*}{2.4} \\
Patch & 0.86 & 0.22 & 0.4 & \\
Monopole & 6.36 & 1.22 & 2.66 & \multirow{2}{*}{5.1} \\
Patch & 0.76 & 0.12 & 0.36 & \\
\hline
\end{tabular}

sorbed power is concentrated in the tissues in the vicinity of the antenna.

The electric field values decrease rapidly as the probe moves toward the centre of the head. The region near the skin-mobile phone interface is therefore primary to characterize.

We begin by comparing the radiation pattern of our patch antenna in a vacuum with that of the phantom presence so we deduce the screen effect applied by the phantom on the normal antenna radiation, Figure 9a shows the radiation pattern of our antenna in a vacuum and Figure $9 \mathrm{~b}$ shows the radiation pattern in the presence of phantom.

It can be clearly seen that the presence of the head has a considerable effect on the radiation pattern of the handset antenna especially in the head direction.

In second place we compare the SAR calculated in the phantom caused by a cell phone equipped with a monopole antenna with that caused by a cell phone equipped with our patch antenna.

Figure 10 shows the SAR quantity caused by the cell phone equipped with monopole antenna compared by the SAR quantity caused by the cell phone equipped with our patch antenna according to the depth in the phantom.

From Table 5, we can clearly see that the skin region is the most exposed, and a large part of the absorbed power is concentrated in the tissues in the vicinity of the antenna. 


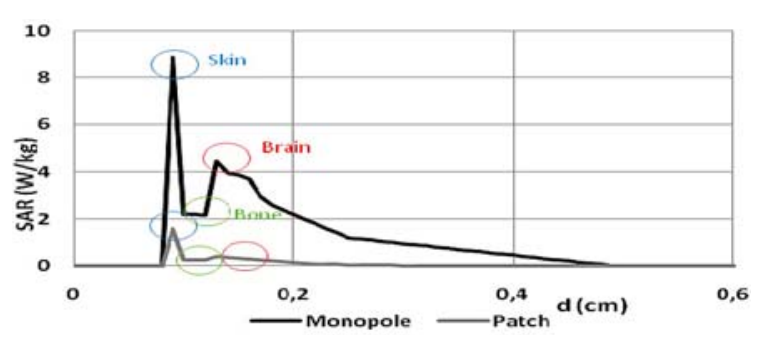

(a)

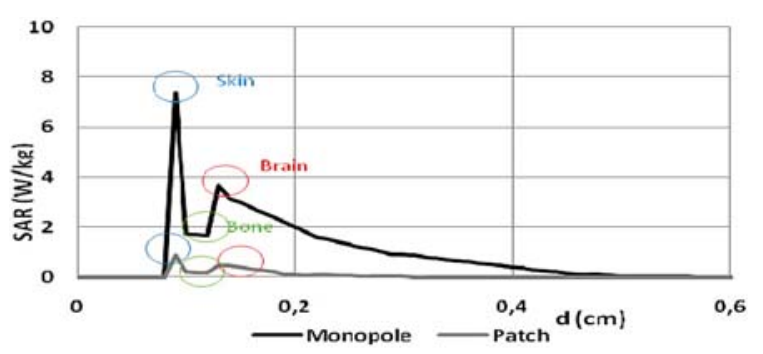

(b)

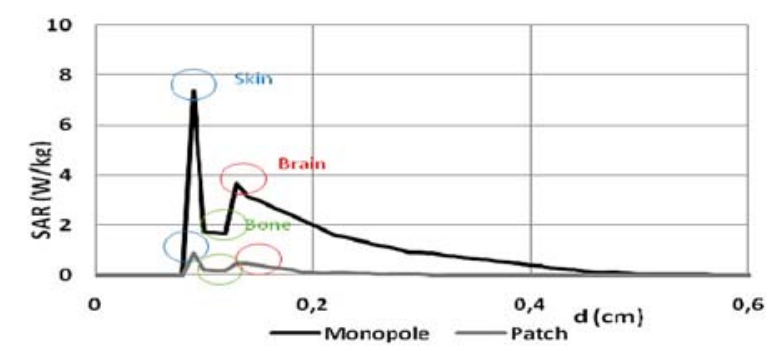

(c)

Fig. 10. (Color online) (a) The SAR quantity in the head caused by the new patch antenna compared with those caused by monopole at $920 \mathrm{MHz}$, (b) the SAR quantity in the head caused by the new patch antenna compared with those caused by monopole at $2.4 \mathrm{GHz}$ and (c) the SAR quantity in the head caused by the new patch antenna compared with those caused by monopole at $5.1 \mathrm{GHz}$.

It has been shown that the SAR peak in the head induced by a multiband patch antenna at the GSM frequency is about $1.6 \mathrm{~W} / \mathrm{kg}$, compared with that induced by a monopole antenna that is approximately $10 \mathrm{~W} / \mathrm{kg}$. The presented antenna respects the SAR limits recommended by the ICNIRP, which is $2 \mathrm{~W} / \mathrm{kg}$ averaged over $10 \mathrm{~g}$ of tissue (Tab. 6).

\section{Conclusions}

A new miniaturized multiband antenna suitable for mobile phone applications and WLAN applications have been
Table 6. SAR limits recommended by ICNIRP.

\begin{tabular}{lcc}
\hline $\begin{array}{l}\text { Exposure } \\
\text { characteristics }\end{array}$ & Frequency range & $\begin{array}{c}\text { Localized } \\
\text { SAR (head } \\
\text { and trunk) } \\
(\mathrm{W} / \mathrm{kg})\end{array}$ \\
\hline Occupational exposure & $100 \mathrm{kHz}-10 \mathrm{GHz}$ & 10 \\
General public exposure & $100 \mathrm{kHz}-10 \mathrm{GHz}$ & 2 \\
\hline
\end{tabular}

presented and studied. Both simulated and measured results have been presented. The studied antenna respects the SAR limits and it present a good radiation in three bands of operation: GSM, Wi-Fi and HiperLan band. A good agreement between the HFSS simulated results and measurements.

This work has been achieved in the setting of the Franco Moroccan cooperation in scientific research and it is done in the IEMN laboratories at USTL Lille.

\section{References}

1. R.A. Sainati, Cad of microstrip antennas for wireless applications (Artech House, Boston, 1996)

2. ICNIP, Health Phys. 74, 494 (1988)

3. IEEE standard for safety levels with respect to human exposure to radio frequency electromagnetic fields, $3 \mathrm{kHz}$ to $300 \mathrm{GHz}$, IEEE Std C95.1 ${ }^{\mathrm{TM}}-2005$ (IEEE, New York, 2005)

4. K.L. Wong, Compact and Broadband Microstrip Antennas (John Wiley \& Sons, Inc., New York, 2002)

5. M. Ben ahmed, M. Bouhorma, F. Elouaai, A. Mamouni, Eur. J. Sci. Res. 32, 151 (2009)

6. S.H. Choi, J.K. Park, S.K. Kim, J.Y. Park, Microw. Opt. Technol. Lett. 40, 399 (2004)

7. A. Kaleghi, M. Kamyab, IEEE Trans. Ant. Propag. 57, $555(2009)$

8. K. Seol, J. Jung, J. Choi, Electron. Lett. 42, 844 (2006)

9. M. Bouhorma, A. Benahmed, F. Elouaai, A. Astito, A. Mamouni, Information and Communication Technologies International Symposium, Tetuan, Morocco, 2005

10. M. Bouhorma, M. Benahmed, F. Elouaai, H. Drissi, A. Mamouni, SAR distribution in the human Head expose to the field radiated by the user antenna of Various Mobile Phone and Wireless LAN antennas, in First Int. Workshop on Wireless Communication in Underground Area, IWWCUCA, Val-d'Or, Quebec, Canada, 2005

11. D. Manteuffel, A. Bahr, D. Heberling, I. Wolff, in Proc. 11th Int. Conf. Ant. Propag., Manchester, UK, 2001, pp. $252-256$ 